\title{
Ein Spinnenkurs für Anfänger und Interessierte
}

\section{Martin Kreuels}

Wer in den letzten Jahren die Teilnehmerzahlen an arachnologischen Jahres- und Regionaltreffen beobachtet hat, wird feststellen können, dass der Boom der Spinneninteressierten aus den 1990er Jahren vorbei ist. Häufiges Problem für Spinneninteressierte ist der Einstieg in unsere Wissenschaft. An den Universitäten fehlt im zunehmenden Maße eine taxonomisch/systematische Ausbildung, nicht nur für die Spinnen. Dass dies nicht nur ein deutsches Problem ist, zeigt ein Artikel der schweizer Kollegen (BURCKHARDT 2005). Neben dem persönlichen Einsatz, der von jedem Einsteiger gefordert ist, um sich einzuarbeiten, ist eine Unterstützung von „erfahrenen“ Arachnologen hilfreich. So lassen sich Probleme schneller erkennen, Kontakte knüpfen und von den langjährigen Erfahrungen profitieren.

Nur woher kommt die Unterstützung? Der eine Weg ist die persönliche Ansprache, um sich einen „Mentor“ zu suchen, der bereit ist, ebenfalls Zeit zu investieren. Dies ist dann ein viel versprechender Ansatz, wenn der Einsteiger dem Ansprechpartner geographisch nahe ist, so dass er beispielsweise an Exkursionen teilnehmen kann. Der andere Weg verläuft über eine öffentliche Veranstaltung (z.B. einen Lehrgang, Workshop etc.), um dort Grundlagen zu erlernen, die dann weiter ausgebaut werden können.

Nachfolgend wird ein Kurs vorgestellt, der diese Einführung bietet:

Die Biologische Station am Rande des Naturschutzgebietes Heiliges Meer ist eine Außenstelle des Westfälischen Museums für Naturkunde in Münster (Nordrhein-Westfalen) und liegt bei Reken/Hopsten im Norden des Bundeslandes. Es bietet im Jahresverlauf zahlreiche taxonomische und systematische Kurse zu Pflanzen-, Pilz- und Tiergruppen an. Die Kurse wenden sich sowohl an Anfänger und Interessierte, als auch an Fortgeschrittene. Von den durchschnittlich 20 (max. 32) Teilnehmern der Kurse stammt eine Hälfte in Regel von der Westfälischen-Wilhelms Universität
Münster und die andere Hälfte sind Interessierte aus allen möglichen Bereichen, die sich sowohl privat, als auch beruflich für das entsprechende Thema interessieren.

\section{Ablauf des Spinnenkurses:}

Der Kurs erstreckt sich über vier Tage (zwei halbe und zwei ganze Tage). Der erste Tag dient der Anreise und einer theoretischen Einführung in das Thema. Die Kurstage zwei und drei sind eine Mischung aus Geländearbeit, Bestimmungen und theoretischen Ergänzungen auf den heterogenen Flächen der Station. Der letzte Tag fasst die Ergebnisse zusammen und beantwortet Fragen der Teilnehmer. Die Unterbringung und Verpflegung findet in der Station statt. Der Vorteil dabei ist, dass auch bis in die späten Abendstunden hinein Diskussionen und weitere Arbeiten stattfinden können.

Ziel des Kurses ist es den Teilnehmern einen ersten Einblick in die Arachnologie zu ermöglichen.

Der Spinnenkurs für Anfänger findet vom 31.7.3.8.2006 zum fünften Mal in Folge statt. Der Preis pro Teilnehmer, ohne Verpflegung, beträgt reduziert $27 €$ und normal $37 €$.

Für weitere Informationen können Interessierte sich an den Leiter der Station NSG 'Heiliges Meer' Dr. Heinrich Terlutter oder an den Autor wenden. Adresse und Kursangebote sind unter: www.heiliges-meer.de zu finden.

\section{Literatur}

BURCKHARDT D. (2005): Bericht und Aktivitäten und Situation der Systematiker in der Schweiz. - GFBS News 14: 20-22

Dr. Martin Kreuels, AraDet, Alexander-HammerWeg 9, 48161 Münster, Tel.: 02533-933545, E-mail: kreuels@bionetworx.de 